\title{
INSTRUMENTO PARA ANÁLISE DE PERIÓDICO DE ENFERMAGEM
}

\author{
Maria Alice Tsunechiro* \\ Daclé Vilma Carvalho** \\ Maria Belén Salazar Posso*** \\ Berenice Xavier Elsas **** \\ Maria do Carmo Lui* \\ Maguida Costa Stefanelli*
}

TSUNECHIRO, M. A.; CARVALHO, D. V.; POSSO, M. B. S.; ElSAS, B. X.; LUI, $M$. do C.; STEFANELLI, M. C. Instrumento para análise de periódico de enfermagem. Rev. Esc. Enf. USP, São Paulo, 17(2):107-117, 1983.

Estudos sobre divulgą̧ão do conhecimento em enfermagem são recentes. Com o objetivo de contribuir para o estudo da produção literária em enfermagem no Brasil, foi elaborado em instrumento para análise de periódicos. $O$ instrumento foi testado em um periódico de enfermagem e foi constatado que ele permite verificar quem escreve, $o$ que escreve $e$ ainda relacionar os trabalhos com a realidade de saúde vigente e com a evolução histórica da profissão.

\section{INTRODUÇÃO}

A produção científica das universidades, segundo RODRIGUEZ (1979), pertence à sociedade, e os resultados de estudos a respeito de seus problemas só tem valor quando comunicados à população pois ao tentar-se uma solução conjunta está-se contribuindo para o desenvolvimento do País. Ainda segundo este autor, as publicações referentes à área de saúde são escassas e a maioria não surge como produto da problemática de saúde dos países; refere que, de maneira semelhante aos processos de formação de recursos humanos em saúde, em particular de recursos médicos, a investigação científica aparece totalmente desvinculada da realidade social, obedecendo às influências externas, da ocasião, totalmente alheias às próprias necessidades dos países.

* Enfermeira. Mestre em Enfermagem. Professor Assistente do Departamento de Enfermagem Materno-Infantil e Psiquiátrica da Escola de Enfermagem da Universidade de São Paulo.

** Enfermeira. Mestre em Enfermagem. Professor Adjunto do Departamento de Enfermagem Básica da Escola de Enfermagem da Universidade Federal de Minas Gerais.

*** Enfermeira. Mestre em Enfermagem. Chefe do Departamento de Enfermagem da Universidade de Taubaté. Professor Adjunto.

**** Enfermeira. Mestre em Enfermagem. Professor Adjunto da Escola de Enfermagem Ana Neri da Universidade Federal do Rio de Janeiro. 
A produção científica da enfermagem no Brasil é matéria que vem preocupando os enfermeiros, principalmente na última década, quando foram despertados para a necessidade de como estruturar o corpo de conhecimento da profissão, melhorar a qualificação dos profissionais, torná-los mais atuantes na sociedade, ampliar seu campo de ação e estabelecer vínculos dos enfermeiros com outros profissionais em equipes de trabalho.

Estes e outros tantos problemas profissionais motivaram seminários e encontros para delimitação de linhas de pesquisa prioritárias e de aplicação na prática profissional.

Estudos a respeito da literatura de enfermagem ainda são escassos em nosso meio. São poucos os livros de enfermagem, em português, e o número de periódicos, no Brasil, de que se tem conhecimnto, não ultrapassa uma dezena - "Revista Brasileira de Enfermagem", "Revista da Escola de Enfermagem da USP", "Revista Gaúcha de Enfermagem", "Enfermagem ein Novas Dimensões" (interrompida em 1979), "Enfoque", "Enfermagem Atual", "Revista Paulista de Enfermagem" e "Enfermagem: O Jornal Brasileiro de Enfermagem".

Por ser reduzido o número de periódicos pode-se afirmar que há pouca divulgação dos conhecimentos desenvolvidos pelos profissionais de enfermagem.

Nos trabalhos de ALMEIDA et alii (1981) e de ANGERAMI \& ALMEIDA (1982) é enfatizada a importância da divulgação do conhecimento científico produzido pelas enfermeiras. Segundo estas autoras, o público a que se destina a publicação a recebe com aplausos ou a rejeita; como profissionais de formação universitária, as enfermeiras têm a responsabilidade de atuar como agente de mudança. Estas autoras consideram inútil a pesquisa não divulgada.

Entre os esforços para estimular a divulgação do conhecimento, evidencia-se a idéia dos membros da Comissão de Pós-Graduação da Escola de Enfermagem da USP em colocar, a partir de 1981, como exame final de qualificação, um trabalho sobre assunto de enfermagem, na forma de artigo para publicação. Tem-se, com isso, dupla finalidade: ensino e estímulo para divulgação do conhecimento.

SECAF (1981), em seu artigo "Vamos escrever para publicar?", incentiva alunas do curso de licenciatura em enfermagem a escreverem para publicação.

As autoras deste trabalho se reportam à contribuição de Maria Rosa Sousa Pinheiro, ex-diretora da Escola de Enfermagem da USP, incansável na luta para estimular enfermeiras a escreverem e pesquisarem. A Revista da Escola de Enfermagem da USP surgiu em 1967 para comemorar os 25 anos de criação da Escola, como resultado do contínuo esforço desta grande mestra da enfermagem para promover o desenvolvimento da profissão. Ainda hoje, aposentada, continua prestar serviços à profissão: é membro da Comissão de Assessoramento Técnico 
da citada revista e fonte de consulta para diversos assuntos profissionais. Ao falar na Revista da Escola de Enfermagem da USP não podemos omitir os nomes de Nahyda de Almeida Velloso e Zuleika Kannebley que tanto trabalharam para a eficácia da mesma.

A preocupação em analisar a literatura de enfermagem é recente.

ALMEIDA et alii (1981) analisam a produção de conhecimento na pós-graduação em enfermagem no Brasil, atendo-se às teses defendidas para obtenção dos graus de mestre, doutor e livre-docente. Concluem que a produção de conhecimento de enfermagem, a nível de pós-graduação, está voltada para os aspectos internos da profissão, não sendo esta enfocada como prática social.

PELA et alii (1981) estudam a situação atual dos cursos e da produção científica da pós-graduação da Escola de Enfermagem de Ribeirão Preto, nos anos de 1978, 1979, e 1980, e consideram satisfatório o resultado da análise. Salientam, ainda, que a produção analisada é resultante de esforços individuais, próprios de uma profissão emergente que ainda não consolidou sua tradição em pesquisa.

Nos trabalhos de VIEIRA (1980); ANDRADE \& NOBREGA (1982); NOGUEIRA (1982); NEVES (s.d.) e nos acima citados (ALMEIDA et alii, 1981 e PELA et alii, 1981) são utilizados esquemas conceituais diferentes para análise da produção literária de enfermagem.

Assim, a fim de contribuir para o estudo da produção literária em enfermagem, o presente trabalho teve como objetivo elaborar e validar um instrumento para análise da produção escrita em periódico de enfermagem.

\section{METODOLOGIA}

\section{INSTRUMENTO.}

Para o estudo da literatura publicada pelos periódicos de enfermagem foram elaborados dois formulários (Anexos I e II), após consulta aos trabalhos de BARNARD \& NEAL (1977), ELLIS (1977), GORTNER \& NAHM (1977), HIGHRITER (1977), SCHLOTFELDT (1977), SILLS (1977), HAYTER \& RICE (1979), VIEIRA (1980), ALMEIDA et alii (1981), PELA et alii (1981), NOGUEIRA (1982), NEVES (s.d.) e discussão em grupo dos autores do presente trabalho.

$O$ instrumento final foi elaborado após estudo preliminar; escolheu-se, para amostra deste, o volume inicial, o intermediário e o último publicado até o momento do estudo, por um periódico nacional de enfermagem. Estes, constituíram $20 \%$ dos volumes já publicados, correspondendo aproximadamente à mesma porcentagem do total dos fascículos.

A anexo I contém informações gerais para caracterizar o periódico. Destaca-se um item que trata da formação profissional dos membros do 
Conselho Editorial; é de suma importância que o mesmo seja representativo da classe profissional a que se destina.

O anexo II contém itens sobre o autor, co-autores e produção literária em si.

Considerou-se como categoria funcional dos autores: professor, auxiliar de ensino, professor assistente, professor adjunto e professor titular, quando sua área de atuação é o de docência. Como grau foram considerados os títulos de mestre, doutor e livre-docente. Na categoria "professor" foram arrolados aqueles autores identificados, em nota de rodapé, como professor, instrutor, docente e professor colaborador, terminologia esta que surge a partir de 1974 em algumas universidades do Brasil.

A produção literária foi classificada segundo campo, área, linha e enfoque, as quais são definidas como segue:

Campo: raio de abrangência de uma profissão. Exemplo: Enfermagem. Ärea: aspecto do campo estudado, analisado ou pesquisado. Exemplo: Administração.

Linha: direcionamento dado à área estudada. Exemplo: Auditoria.

Enfoque: será usado no presente estudo apenas para identificar se publicação reflete aspecto hospitalar, comunitário ou ambos.

Para análise da literatura dividiu-se esta em: 1. artigos de divulgação científica; 2. relatos de pesquisa e 3 . outros.

1. A produção denominada artigo de divulgação científica foi estudada em relação à área abordada e enfoque. As publicações foram agrupadas nas seguintes áreas:

1.1. assistência;

1.2. ensino;

1.3. profissão;

1.4. administração;

1.5. metodologia de pesquisa; e

1.6. outras.

1.1. Na área assistência foram incluídos os artigos relacionados às seguintes linhas:

- sistematização da assistência de enfermagem;

— planejamento da assistência;

- fundamentação para assistência;

- necessidades ou problemas do paciente; e

- educação para a saúde. 
Em sistematização da assistência de enfermagem foram incluídos os artigos referentes a métodos de assistência e modelos de planejamento de assistência.

Planejamento da assistência abrange levantamentos de dados sobre o paciente, diagnóstico de enfermagem, plano de cuidados ou recomendações de enfermagem, execução do plano ou das recomendações, avaliação destes e relacionamento enfermeira-paciente. Procurou-se colocar aqui as diferentes denominações dadas aos passos da assistência de enfermagem, para atender à terminologia usada, desde o início da publicação da revista.

Fundamentos para assistência de enfermagem integram todas as publicações que oferecem conhecimentos científicos para o desenvolvimento da assistência de enfermagem.

Necessidades e problemas do paciente incluem publicações que abordam necessidades psicobiológicas, psicossociais e psicoespirituais.

Educação para a saúde aborda as publicações referentes ao auto-cuidado, educação sanitária e orientação da população sobre aspectos de saúde.

1.2. As publicações relativas à área de ensino foram agrupadas nas seguintes linhas:

- currículo (englobando legislação, filosofia, conceito, modelo, plano de curso ou disciplina e carga horária);

- avaliação do processo ensino-aprendizagem;

- métodos e técnicas de ensino;

- estratégias de ensino em enfermagem;

- integração docente-assistencial;

- caracterização do aluno;

- recursos humanos e materiais.

1.3. Na área administração as publicações ficaram arroladas nas linhas a seguir: toria);

- funções da enfermeira (incluindo-se supervisão, liderança e audi-

- filosofia de atendimento ao cliente;

- recursos humanos e materiais;

- educação continuada;

- educação em serviço;

- trabalho em equipe. 
1.4 A área profissão teve as publicaçôes pertinentes agrupadas nas seguintes linhas:

— história e legislação;

- deontologia;

- teorias de enfermagem;

- enfermagem como prática social;

- filosofia e valores profissionais;

- perfil do enfermeiro;

- associação de classe.

1.5. A área metodologia de pesquisa abrange as publicações referentes às linhas:

- fundamentação teórica sobre pesquisa em enfermagem;

- instrumentos e técnicas para pesquisa em enfermagem.

Esta área foi estudada separadamente por ser considerada de fundamental importância para uma profissão que está desenvolvendo seu corpo próprio de conhecimento.

1.6. Em outras áreas foram arroladas todas as publicações não passiveis de agrupamento nas áreas já descritas.

Quanto ao enfoque o limitamos somente a: hospitalar, comunitário e ambos (hospitalar e comunitário) uma vez que as linhas, direção dada ao assunto, estão descritas nos agrupamentos das áreas.

2. No estudo das publicações referentes a relatos de pesquisa obedeceu-se à mesma denominação empregada no estudo de artigos.

Foram acrescentados itens para verificar se a pesquisa foi realizada para obtenção ou não de grau, para facilitar o estudo sobre a correlação com o desenvolvimento histórico da profissão e de pesquisa em enfermagem.

3. No item "outros" foram agrupados eventos, editoriais, palestras, discursos, conferências, notas prévias, sendo que estes foram estudados só do ponto de vista quantitativo e em relação a seus autores e respectiva formação profissional.

\section{PROCEDIMENTOS PARA VALIDAÇÃO}

Para preenchimento do formulário foram usadas somente as informações contidas no periódico, na própria publicação - capa e contra-capa - e nos editoriais. 
Para preenchimento do formulário assegurou-se sempre a presença de três c'crinentos do grupo (total de 6) responsáveis pelo estudo, para garantir uniĩrinidade na colheita dos dados.

Cada publicação foi estudada por meio da leitura do resumo, quando havia, e de uma leitura da sua totalidade; quando necessário, procedia-se à leitura pormenorizada da mesma. Só então, era feito o preenchimento do formulário.

Quando havia alguma dúvida sobre como considerar o dado esta era discutida na presença de todos os elementos do grupo.

A validação foi realizada analisando-se todos os volumes, já publicados do periódico utilizado para o estudo.

\section{COMENTARIOS}

A avaliação do instrumento permite inferir que este pode ser aplicado a qualquer periódico de enfermagem. $O$ instrumento permitiu colher dados, entre outros aspectos, sobre: quem está escrevendo - enfermeira docente ou de campo, se há integração entre estas, para escreverem artigos, se possuem grau acadêmico ou não, como estão as pesquisas sendo realizadas, por quem, qual a área, a linha e o enfoque das mesmas e ainda, sobre a evolução da profissão. Se com a utilização do instrumento foi possivel identificar estes dados pode-se dizer que o periódico retrata o campo - a enfermagem.

Pela análise dos dados obtidos no instrumento proposto foi possível perceber a evolução histórica da enfermagem, correspondente ao período da publicação do periódico estudado e, em parte, dos períodos antecedentes. Esta evolução pode ser percebida, por exemplo, quando surgem vários artigos e simpósio sobre currículo, logo após a reforma universitária no País. Outro exemplo, é o aparecimento de publicação de pesquisas, em sua maioria para obtenção de grau de mestre ou doutor. Outros trabalhos, frutos de pesquisa ou não, refletem a preocupação com a qualidade da assistência de enfermagem, com a conceituação da enfermagem e com a enfermagem como ciência e profissão.

O aumento de pesquisas surge após a reforma universitária e com a conseqüente criação dos cursos de mestrado.

Outro fato interessante, que chamou a atenção das autoras, foi o aumento de citações de autores nacionais, pelo menos em parte das publicações.

\section{SUGESTŐES}

A análise feita para validação do instrumento permite as sugestões que seguem.

1. Além da composição do Conselho Editorial, deve constar a formação profissional de cada um de seus elementos. Isto é de suma importância, pois este deve ser composto por elementos que representem a classe profissional a que se destina o periódico. 
2. A indexação, nivel nacional e internacional, das publicações do periódico, se houver, deve constar junto às outras informaçōes gerais sobre o mesmo.

3. Toda publicação deve apresentar um resumo, em pelo menos duas línguas - português e inglês no nosso caso - para facilitar indexação e permuta. Este resumo deve dar as linhas gerais do trabalho apresentado: o problema do estudo, objetivo deste e os principais tópicos abordados no mesmo.

4. As informações sobre o autor e co-autores devem apresentar, em primeiro lugar, qual a formação profissional, seguida de grau acadêmico, categoria profissional, instituição a que está vinculado e outros títulos, se houver.

5. Os resumos de monografias e teses devem conter o problema em estudo, os objetivos, a metodologia bem descrita e as conclusões para que dêem uma noção mais exata do trabalho.

Os autores deste trabalho esperam que sejam enviadas sugestões e críticas sobre o instrumento apresentado e terminam este citando Marina de Andrade RESENDE (1963).

"Contribuir para a expansão do conhecimento científico em linguagem técnica é uma atribuição a que os enfermeiros não se podem furtar; e uma responsabilidade que não pode ser delegada às outras categorias profissionais; exige, por isso mesmo, reflexão constante e progressiva, sem o que estarão os enfermeiros omitindo um dever".

Para aquelas que alegam não ter tempo para divulgar o seu saber, a citada autora chama atenção para:

"As enfermeiras, mesmo as que sabem e podem escrever, são muito ocupadas; entretanto, a ocupação e a obrigação, compreendidas, e aceitas afetiva e inteligentemente, geram a ordem e assim, algumas de nossas colegas souberam encontrar tempo para enriquecer o patrimônio de enfermagem com artigos e livros, abrindo caminhos a serem trilhados com mais freqüência e menos dificuldades pelas gerações mais jovens".

TSUNECHIRO, M. A.; CARVALHO, D. V.; POSSO, M. B. S.; ElSAS, B. X.; LUI, $M$. do $C$.; STEFANELLI, M. C. Instrument to analyse periodicals of nursing. Rev. Esc. Enf. USP, São Paulo, $17(2): 107-117,1983$.

Studies about spreading of knowledge in nursing are recent. In order to contribuite to the study of the nursing literature in Brazil, it was developed a model of instrument to analyse periodicals. The instrument was tested in a nursing periodical and it was found to be effective in stablishing who writes, what is written and even to relate the papers with present state of health and the historical evolution of the profession. 


\section{REFERENCIAS BIBLIOGRAFICAS}

ALMEIDA, M. C. P. de et alii. A produção do conhecimento na pós-graduação em enfermagem no Brasil. In: CONGRESSO BRASILEIRO DE ENFERMAGEM. 33\%, Manaus, 2-8 de agosto, 1981.

ANDRADE, M. N. de \& NoBREGA, M. R. S. Núcleos emergentes de pesquisa e pós-graduação em enfermagem. In: SEMINARIO de Pesquisa em Enfermagem: avaliação e perspectivas, 24 Brasilia, 24-26 de março, 1982. 21 p.

ANGERAMI. E. L. S. \& ALMEIDA, M. C. P. de Divulgação do conhecimento científico produzido na enfermagem. 1982. (mimeografado).

BARNARD, K. E. \& NEAL, M. V. Maternal-child nursing research: review of the past and strategies for the future. Nurs. Res., New York, 26(3):193-200, May/June, 1977.

ELLIS, R. Fallibilities, fragments and frames: contemplation on 25 years of research in medical-surgical nursing. Nurs. Res., New York, 26(3):177-82, May/June, 1977.

HAYTER, J. \& RICE, P. Institutional sources of articles. Nurs. Res., New York, 28(4):205-9, July/Aug. 1979.

HIGHRITER, M. E. The status of community health nursing research. Nurs. Res., New York, 26(3):183-92, May/June 1977.

GORTNER, S. R. \& NAHM, H. An overview of nurging research in the United States. Nurs. Res., New York, 26(1):19-33, Jan./Feb. 1977.

NEVES, E. P. Vazios do conhecimento e sugestões de temáticas relevantes na área da enfermagem. (mimeografado).

NOGUEIRA, M. J. de C. A pesquisa em enfermagem no Brasil: retrospectiva histórica. Rov. Esc. Ene. USP, São Paulo, 16(1):17-26, abr. 1982.

PELA, N. T. R. et alii. Situação atual dos cursos e produção cientifica da pós-graduação da Escola de Enfermagem de Ribeirão Preto (1978, 1979, 1980). 1981. (mimeografado).

RESENDE, M. de A. Literatura profissional de enfermagem. Bev. Bras. Enf., Rio de Janeiro, 16(2) :133-40, fev. 1963.

RODRIGUEZ, M. I. El trabajo científico en la formación de profissionales de salud. Educ. Mod. Salud, Washington, 13(3):212-229, 1979.

SCHLOTFELDT, R. M. Nursing research: refletion of values. Nurs. Ros., New York, 26(1) :4-9, Jan./Feb. 1977.

SECAF, V. Vamos escrever para publicar? (Uma estratégia de ensino). Rev. Esc. Ene. USP, São Paulo, 15(1):55-62, abr. 1981.

SILLS, G. M. Research in the fleld of psychiatric nursing 1952-1977. Nurs. Res., New York, 26(3):201-7, May/June 1977.

VIEIRA, T. T. Produção cientifica em enfermagem no Brasil 1960-1979. Salvador, 1980. (Tese - Professor Titular. Universidade Federal da Bahia). $200 \mathrm{p}$. 


\section{CARACTERIZAÇAO DO PERIODICO}

Nome:

Local de Publicação:

Início da Publicação:

Periodicidade:

Finalidade da Criação da Revista:

Público a que se Destina:

Intercâmbio:

Redator-Chefe:

Formação Profissional:

Conselho Editorial:

Número de Membros:

Formação Profissional dos Membros:

Normas para Publicação:

Index: Anual

Cumulativo:

Indexação Nacional:

Internacional:

Esquema do Conteúdo:

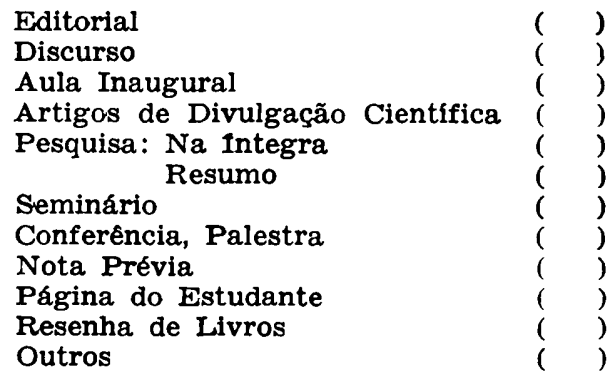

Publica:

Tabelas: $\quad$ Gráficos:

Figuras:

Ilustraçōes:

Anexos:

Resumo dos Trabalhos:

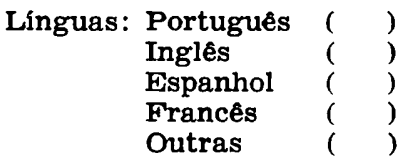

Bibliografia:

Referências Bibliográficas:

Capa (descrição sucinta):

Tiragem: 
1. Ano: 19..

2. Volume: .....

3. $\mathrm{N} \cdot \ldots$.

4. p. ....

5. Titulo:

6. Categoria Profissional ou Ocupacional do Autor:

7. Area de Atuação do Autor:

7.1 Docência

7.1.1 Categoria Funcional:

7.1.2 Grau:

7.1.3 Instituição e Local:

7.2 Assistência:

7.2.1 Categoria Funcional:

7.2.2 Grau:

7.2.3 Instituição e Local:

7.3 Outra:

7.3.1 Categoria Funcional:

7.3.2 Grau:

7.3.3 Instituição e Local:
8. Co-Autores:

8.1 Número:

8.2 Categoria Profissional ou Ocupacional:

9. Artigo de Divulgação Científica

9.1 Campo:

9.2 Ârea:

9.2.1 Assistência

9.2.2 Ensino

9.2.3 Profissão

9.2.4 Administração

9.2.5 Metodologia de Pesquisa

9.2.6 Outra

9.3 Linha:

9.4 Enfoque: Hospitalar ( ) Comunitário ( ) Ambos ( )

10. Relato de Pesquisa

10.1 Publica: Na Integra

10.2 Obtenção de Grau: Sim

$\begin{array}{lrll}( & \text { ( ) } & \text { Resumo } & ( \\ \text { ( } & \text { Não } & (\end{array}$

10.3 Campo:

10.4 Area:

10.4.1 Assistência

10.4.2 Ensino

10.4.3 Profissão

10.4.4 Administração

10.4.5 Outra

10.5 Linha:

10.6 Enfoque: Hospitalar ( ) Comunitário ( ) Ambos ( )

11. Outros: Editorial ( ) Conferência, Palestra ( ) Nota Prévia

Discurso ( ) Resenha de Livros ( ) Outras Informações

12. Observações do Pesquisador: 